\title{
Characterization of disease course and remission in early seropositive rheumatoid arthritis: results from the TACERA longitudinal cohort study
}

Ther Adv Musculoskel Dis 2021, Vol. 13: 1-17 DOI: $10.1177 /$ $1759720 \times 211043977$

(c) The Author(s), 2021. Article reuse guidelines: sagepub.com/journalspermissions

\section{RA-MAP Consortium}

\begin{abstract}
Background: To characterise disease course and remission in a longitudinal observational study of newly diagnosed, initially treatment-naïve patients with seropositive rheumatoid arthritis (RA).

Methods: Patients with early untreated seropositive RA were recruited from 28 UK centres. Multiple clinical and laboratory measures were collected every 3 months for up to 18 months. Disease activity was measured using the 28 -joint Disease Activity Score with C-reactive protein (DAS28-CRP) and Simplified Disease Activity Index (SDAI). Logistic regression models examined clinical predictors of 6 -month remission and latent class mixed models characterised disease course.
\end{abstract}

Results: We enrolled 275 patients of whom 267 met full eligibility and provided baseline data. According to SDAl definition, 24.3\% attained 6-month remission. Lower baseline Health Assessment Questionnaire (HAQ) and SDAI predicted 6-month remission ( $p=0.013$ and 0.011 ). Alcohol intake and baseline prescribing of methotrexate with a second disease-modifying antirheumatic drug (DMARD; vs monotherapy without glucocorticoids) were also predictive. Three distinct SDAl trajectory subpopulations emerged; corresponding to an inadequate responder group $(6.5 \%)$, and higher and lower baseline activity responder groups $122.4 \%$ and 71.1\%). Baseline HAQ and Short Form-36 Health Survey - Mental Component Score (SF$36 \mathrm{MCS}$ ) distinguished these groups. In addition, a number of baseline clinical predictors correlated with disease activity severity within subpopulations. Beneficial effects of alcohol intake were found across subpopulations.

Conclusion: Three distinct disease trajectory subpopulations were identified. Differential effects of functional and mental well-being, alcohol consumption, and baseline RA medication prescribing on disease activity severity were found across subpopulations. Heterogeneity across trajectories cannot be fully explained by baseline clinical predictors. We hypothesise that biological markers collected early in disease course (within 6 months) may help patient management and better targeting of existing and novel therapies.

Keywords: disease activity, latent class-mixed models, remission, rheumatoid arthritis, SDAI trajectories

Received: 1 February 2021; revised manuscript accepted: 17 August 2021

\section{Introduction}

Early diagnosis and prompt, tight control of disease activity have been proven to alter long-term course of rheumatoid arthritis (RA) and limit structural damage and long-term disability. ${ }^{1}$ In spite of a range of synthetic and biological disease-modifying antirheumatic drugs (DMARDs), clinical remission is achieved and sustained in a
Correspondence to: Brian Tom MRC Biostatistics Unit University of Cambridge, Cambridge CB2 OSR, UK. brian.tomamrc-bsu.cam .ac.uk

Andrew Cope

Academic Department of

Rheumatology, Division

of Immunology and

Inflammatory Diseases,

Faculty of Life Sciences, King's College London,

London, UK.

andrew.copelakcl.ac.uk 
minority, and drug-free remission remains rare. ${ }^{2}$ This may be due to many factors. For example, there are no validated instruments for reliably predicting prognosis. Nor is it possible to confidently predict which patients will respond more favourably to one particular drug or drug combination over another. Furthermore, our understanding of low disease activity states is limited. ${ }^{3,4}$ The ability to employ the most appropriate 'treatto-target strategy' to the right set of early RA patients would be a major advance. Recent evidence suggests that for early RA patients following a treat-to-target strategy, distinct trajectories of disease activity over the first year exist. ${ }^{5}$ Moreover, evidence from long-term observational cohorts has suggested that distinct trajectories linked to prognosis exist; which may point to distinct immunopathogenic subsets. ${ }^{6}$ Even anticitrullinated protein antibody (ACPA)-positive disease is heterogeneous in outcomes (e.g. radiological progression), further adding to arguments favouring a stratified medicine approach. 7,8

To facilitate the goals of the RA MRC/ABPI (RA-MAP) Consortium, ${ }^{9}$ a cohort of newly diagnosed seropositive RA patients - the 'Towards A CurE for RA' (TACERA) cohort - was established and followed frequently and deeply phenotyped. The aim of this study was two-fold. First, we aimed to determine if baseline clinical factors and RA-prescribed medications are associated with 6-month remission in seropositive early RA. The 6-month time point was chosen to evaluate the primary outcome of remission as this corresponds to when therapy escalation would generally be considered following NICE guidelines. However, an analysis at a single time point neither reflects longitudinal disease activity patterns nor what factors predict, for example, sustained remission, fluctuating disease activity course, or gradual versus rapid response. Therefore second, we aimed to (1) determine whether different types of disease activity trajectory subpopulations exist within this inception cohort; (2) identify factors associated with longitudinal disease activity; and (3) determine whether differences in trajectory types (if they exist) associate with disease outcomes, baseline RA-prescribed medication, smoking status, and alcohol consumption. Identification of factors associated with disease activity and characterization of subgroups may improve patients' treatment and management and identify the most suitable patients for recruitment into trials.

\section{Methods}

\section{Patients}

We recruited newly diagnosed patients $\geqslant 18$ years of age with symptom duration $<12$ months who fulfilled either the 1987 American College of Rheumatology (ACR) or 2010 European League Against Rheumatism (EULAR)/ACR classification criteria for RA. ${ }^{10,11}$ Patients were required to be ACPA and/or rheumatoid factor (RF) positive and naïve to DMARDs or glucocorticoid therapy. In addition, for patients recruited, there needed to be a clear intention by the supervising rheumatologist to commence therapy with DMARDs. Patients were excluded if they had significant comorbidities or if pregnant or wishing to conceive. Participation in trials impacting on patient's treatment, immune status, or disease activity during the study period was not permitted. Ethical approval was authorised by the National Research Ethics Service London Central Committee (Reference number: 12/LO/0469). Informed, written consent was obtained.

\section{Study design}

Subjects were recruited from 28 UK centres. Study sample size was determined as described in the Supplementary Material. Following enrolment, subjects received treatment using conventional synthetic DMARDs (csDMARDs), with therapy adjustments made at the supervising rheumatologist's discretion according to the National Institute for Health and Clinical Excellence (NICE) guidelines for RA management in adults. ${ }^{12}$ Patients were followed for a period of 18, 12, or 6 months, dependent on time of enrolment (i.e. pre $01 / 07 / 14$, $01 / 07 / 14-31 / 12 / 14$ and post 31/12/14), and seen quarterly for scheduled assessments. Clinical, laboratory, lifestyle, medication, patient-reported outcome measures (PROMs), extra-articular RA features, adverse events, and biological samples were collected at visits. In addition, radiographs of the hands and feet were performed at baseline and 12 months or baseline and 6 months for subjects entering within the third enrolment period.

\section{Biological treatment}

Subjects with inadequate clinical responses to csDMARDs and persistent high disease activity 
(DAS28 $>5.1$ ) at 6 months could receive biological DMARDs (bDMARDs), according to NICE guidelines.

\section{Outcome measures}

The co-primary outcomes were disease remission at 6 months and repeated disease activity over time. Disease activity was measured using both composite 28-joint Disease Activity Score with C-reactive protein, DAS28-CRP (4-component), and Simplified Disease Activity Index (SDAI) scores. ${ }^{13-16}$ Disease remission was defined using both DAS28-CRP remission criterion (DAS28$\mathrm{CRP}<2.6)$ and the more stringent SDAI criterion $(\mathrm{SDAI} \leqslant 3.3)$.

Secondary outcome measures included the ACR/ EULAR Boolean definition of remission, ${ }^{17}$ 28-joint Disease Activity Score with erythrocyte sedimentation rate (DAS28-ESR), annualised rate of radiographic damage progression as measured by modified Larsen's score, ${ }^{18-20}$ functional disability as measured by the Health Assessment Questionnaire (HAQ), ${ }^{21}$ and quality of life using both the Medical Outcomes Study Short Form36 Health Survey (SF-36) and EuroQoL five dimensions questionnaire (EQ-5D).22-24

\section{Statistical methods}

Baseline and 6-month information were summarised using the mean, with accompanying standard deviation (SD) for continuous variables, while binary or categorical variables were summarised using frequency and percentage.

To identify baseline predictors of 6-month clinical remission, a two-stage approach was adopted. In the first stage, variables, previously identified from the RA literature ${ }^{25-30}$ as potential predictors of remission, were univariately screened (using univariate logistic regression models with a conservative screen positive $p$ value threshold of $p<0.2$ ). Those screened positive in the first stage were taken forward to the second stage and included in multivariate logistic regression models that additionally included baseline prescribing of (or baseline intention to prescribe and then administered within 3 months) RA medication. Considered predictors in the first-stage comprised age, sex, ethnicity, body mass index (and obesity), symptom duration, smoking status, baseline disease activity, HAQ, SF-36 Mental Component Score (SF-36
MCS), alcohol consumption, serology ( $R F$ and ACPA), smoking, and erosion.

Latent class mixed models (LCMMs) were used to cluster disease activity trajectories that may identify clinically important subpopulations and characterise disease activity longitudinally. Within each latent class, fixed and random patient-level intercepts and piecewise linear time effects (at 5 months) were considered for the linear mixed-model part along with potential predictors informed from the earlier analyses on clinical remission. No covariates were included in the class-membership model part. LCMMs are likelihood-based methods, which are valid using only observed data, under a missing-atrandom assumption. Associations of latent trajectory classes with outcomes, baseline RA medication prescribing, alcohol and smoking status were assessed either using analysis of variance or Fisher's exact test. For the purpose of these association tests and more generally, patients were hard assigned to a particular latent trajectory class based on a posterior classification of class membership through the selection of the patient's class with the highest estimated posterior class-membership probability. The best choice of the number of latent classes (3 classes vs 4 classes) was made using Bayesian Information Criterion (BIC) and relative entropy.

Statistical analyses were performed using the base library glm function for logistic regression and the hlme function in the $1 \mathrm{cmm}$ package in the $R$ statistical software. ${ }^{31}$ Two patients, not prescribed RA medications within the first 3 months, were excluded from the regression analyses.

The reporting of this study conforms to the Strobe statement. ${ }^{32}$

\section{Results}

\section{Patient characteristics}

Two hundred and seventy-five patients were recruited of whom 270 fulfilled all eligibility criteria. Two eligible patients withdrew at baseline without providing any clinical information. A further patient who withdrew at baseline had some clinical information but insufficient to calculate disease activity scores. Table 1 summarises the baseline characteristics of the remaining 267 patients. Briefly, the mean age at entry was 53.1 (SD of 15.2 ); $72 \%$ were female, $72.7 \%$ white, $31.8 \%$ healthy weight and the mean Charlson's Comorbidity Index (CCI), 33 
Table 1. Baseline characteristics of patients in the TACERA study.

\begin{tabular}{|c|c|c|}
\hline Characteristics & $\begin{array}{l}\text { Baseline } \\
(n=267)\end{array}$ & $\begin{array}{l}\text { Missing } \\
\text { data } \\
\text { frequency }\end{array}$ \\
\hline Age, years & $53.1(15.2)$ & 0 \\
\hline Female & $192(71.9 \%)$ & 0 \\
\hline White ethnicity & $194(72.7 \%)$ & 0 \\
\hline BMI & & 0 \\
\hline Female & $27.53(6.47)$ & \\
\hline Male & $27.43(4.97)$ & \\
\hline Overall & $27.50(6.08)$ & \\
\hline BMI status & & 0 \\
\hline Underweight: $<18.5$ & $9(3.4 \%)$ & \\
\hline Healthy weight: $(18.5,25)$ & $85(31.8 \%)$ & \\
\hline Overweight: $(25,30)$ & $95(35.6 \%)$ & \\
\hline Obese: $\geqslant 30$ & $78(29.2 \%)$ & \\
\hline Smoking & & 0 \\
\hline Never smoked & $95(35.6 \%)$ & \\
\hline Previous smoker & $104(39.0 \%)$ & \\
\hline Current smoker & $68(25.4 \%)$ & \\
\hline Alcohol consumption & & 1 \\
\hline None & $86(32.3 \%)$ & \\
\hline $1-5$ units per week & $115(43.2 \%)$ & \\
\hline $6-10$ units per week & $25(9.4 \%)$ & \\
\hline $11-15$ units per week & $11(4.1 \%)$ & \\
\hline $16-20$ units per week & $15(5.7 \%)$ & \\
\hline $\begin{array}{l}\text { More than } 20 \text { units per } \\
\text { week }\end{array}$ & $14(5.3 \%)$ & \\
\hline Alcohol frequency & & 0 \\
\hline Not drinking & $86(32.2 \%)$ & \\
\hline $1-2$ days a year & $28(10.5 \%)$ & \\
\hline $1-2$ days a month & $48(18.0 \%)$ & \\
\hline $1-2$ days a week & $58(21.7 \%)$ & \\
\hline $3-4$ days a week & $28(10.5 \%)$ & \\
\hline 5 days or more a week & $19(7.1 \%)$ & \\
\hline
\end{tabular}

Table 1. (continued)

\begin{tabular}{|c|c|c|}
\hline Characteristics & $\begin{array}{l}\text { Baseline } \\
(n=267)\end{array}$ & $\begin{array}{l}\text { Missing } \\
\text { data } \\
\text { frequency }\end{array}$ \\
\hline RF positive & $247(92.5 \%)$ & 0 \\
\hline ACPA positive & $230(86.1 \%)$ & 0 \\
\hline Disease duration (years) & $0.43(0.23)$ & 0 \\
\hline $\begin{array}{l}\text { X-ray Larsen's Score } \\
\text { (hands and feet) }\end{array}$ & $6.70(8.76)$ & 6 \\
\hline $\begin{array}{l}\text { Charlton's Comorbidity } \\
\text { Index (original) }\end{array}$ & $0.44(0.84)$ & 0 \\
\hline $\begin{array}{l}\text { Charlton's Comorbidity } \\
\text { Index (2008) }\end{array}$ & $0.81(1.10)$ & 0 \\
\hline SDAI & $28.80(14.29)$ & 3 \\
\hline DAS28-CRP & $4.85(1.22)$ & 2 \\
\hline Prescribed medication & & 0 \\
\hline Methotrexate (MTX) & $202(75.7 \%)$ & \\
\hline Hydroxychloroquine & $141(52.8 \%)$ & \\
\hline Leflunomide & $0(0.0 \%)$ & \\
\hline Sulfasalazine & $18(6.7 \%)$ & \\
\hline Oral glucocorticoids & $17(6.4 \%)$ & \\
\hline $\begin{array}{l}\text { Parenteral } \\
\text { glucocorticoids }\end{array}$ & $126(47.2 \%)$ & \\
\hline No RA medication & $2(0.7 \%)$ & \\
\hline $\begin{array}{l}\text { Medication combinations } \\
\text { prescribed }\end{array}$ & & 0 \\
\hline No RA medication & $2(0.7 \%)$ & \\
\hline MTX only & $51(19.1 \%)$ & \\
\hline Other DMARDs only & $20(7.5 \%)$ & \\
\hline Oral glucocorticoids only & $2(0.7 \%)$ & \\
\hline $\begin{array}{l}\text { Parenteral } \\
\text { glucocorticoids only }\end{array}$ & $15(5.6 \%)$ & \\
\hline MTX and other DMARDs & $53(19.9 \%)$ & \\
\hline $\begin{array}{l}\text { MTX and oral } \\
\text { glucocorticoids }\end{array}$ & $6(2.2 \%)$ & \\
\hline $\begin{array}{l}\text { MTX and parenteral } \\
\text { glucocorticoids }\end{array}$ & $33(12.4 \%)$ & \\
\hline $\begin{array}{l}\text { Other DMARDs and oral } \\
\text { glucocorticoids }\end{array}$ & $2(0.7 \%)$ & \\
\hline $\begin{array}{l}\text { Other DMARDs } \\
\text { and parenteral } \\
\text { glucocorticoids }\end{array}$ & $23(8.6 \%)$ & \\
\hline
\end{tabular}


Table 1. (continued)

\begin{tabular}{|c|c|c|}
\hline Characteristics & $\begin{array}{l}\text { Baseline } \\
(n=267)\end{array}$ & $\begin{array}{l}\text { Missing } \\
\text { data } \\
\text { frequency }\end{array}$ \\
\hline $\begin{array}{l}\text { Oral and parenteral } \\
\text { glucocorticoids }\end{array}$ & $1(0.4 \%)$ & \\
\hline $\begin{array}{l}\text { MTX, other DMARDs } \\
\text { and oral glucocorticoids }\end{array}$ & $5(1.9 \%)$ & \\
\hline $\begin{array}{l}\text { MTX, other DMARDs } \\
\text { and parenteral } \\
\text { glucocorticoids }\end{array}$ & $53(19.9 \%)$ & \\
\hline $\begin{array}{l}\text { MTX, oral and parenteral } \\
\text { glucocorticoids }\end{array}$ & $1(0.4 \%)$ & \\
\hline Medication pyramid & & 0 \\
\hline No RA medication & $2(0.7 \%)$ & \\
\hline MTX only & $51(19.1 \%)$ & \\
\hline $\begin{array}{l}\text { Other DMARDs without } \\
\text { glucocorticoids }\end{array}$ & $73(27.4 \%)$ & \\
\hline $\begin{array}{l}\text { Glucocorticoids with/ } \\
\text { without other RA } \\
\text { medication }\end{array}$ & $141(52.8 \%)$ & \\
\hline \multicolumn{3}{|c|}{$\begin{array}{l}\text { ACPA, anticitrullinated protein antibody; BMI, body mass } \\
\text { index; DAS28-CRP, 28-joint Disease Activity Score with } \\
\text { C-reactive protein; MTX, methotrexate; RA, rheumatoid } \\
\text { arthritis; RF, rheumatoid factor; SDAI, Simplified Disease } \\
\text { Activity Index; TACERA, Towards A CurE for RA. } \\
\text { Values are number (percentage) or mean (standard } \\
\text { deviation). }\end{array}$} \\
\hline
\end{tabular}

modified to exclude rheumatic disease as this applied to all subjects, was 0.44 (SD of 0.84), conforming to the intention of not recruiting patients with significant comorbidities.

Of the 267 patients, 130 (48.7\%), 67 (25.1\%), and $70(26.2 \%)$ were enrolled in the first, second, and third recruitment periods, respectively. No statistically significant differences were found across these three groups with respect to diseaserelated variables, prescribed medication, and PROMs at baseline. However, there were statistically significant differences found in the age, ethnicity, alcohol consumption, and CCI distributions, with the third group being the oldest on average ( 51.5 vs 53 vs 56 years old), having the highest proportion of whites $(71.5 \%$ vs $62.7 \%$ vs $84.3 \%)$, lowest proportion not consuming alcohol at entry $(32.3 \%$ vs $44.8 \%$ vs $20 \%)$ and lowest levels of comorbidities (CCIs of 0.52 vs 0.49 vs 0.26 ).
Overall, patients had moderate to severe disease at baseline as measured by both DAS28-CRP and SDAI. After baseline assessment, methotrexate (MTX) was prescribed to $75.7 \%$ of patients; $58.4 \%$ were prescribed nonmethotrexate major DMARDs $(51.7 \%$ hydroxychloroquine alone, $5.7 \%$ sulfasalazine alone, and $1.1 \%$ both hydroxychloroquine and sulfasalazine); $6.4 \%$ received oral glucocorticoids (average prednisolone dose of $11.1 \mathrm{mg} /$ day, range $=4-30 \mathrm{mg} /$ day) and $47.2 \%$ parenteral glucocorticoids (i.e. intra-articular, intravenous, or intramuscular glucocorticoid administration). Overall, 33\% were prescribed only one class/type of medication, while $66.3 \%$ were prescribed combination therapy (including with glucocorticoids) at baseline. Based on medication patterns at baseline, $26.6 \%, 19.9 \%$, and $52.8 \%$ were prescribed single-RA therapy excluding glucocorticoids, dual therapy (i.e. MTX with another DMARD excluding glucocorticoids), and therapies that included glucocorticoid usage, respectively. Initial pattern of therapy did not appear to associate with either baseline X-ray scores or CCI ( $p=0.56$ and 0.23 , respectively) but, as expected, was associated with disease activity measured using both SDAI and DAS28CRP ( $p=0.01$ and 0.004 , respectively). Two subjects did not receive any RA medication by the time of their first follow-up assessment and were excluded from analyses.

\section{Disease activity, response, and remission \\ at 6 months}

Two hundred and forty-five patients (of the 267) were followed up to or beyond their 6-month assessment visit, with $75,2,60,3$, and 105 having their last assessment visit at $6,9,12,15$, and 18 months, respectively. Of those recruited in the first, second, and third periods, $80.8 \%$ (105), $83.6 \%(56)$ and $92.9 \%(65)$ reached their target follow-up assessment visits of 18, 12, and 6 months, respectively. Of the 245 followed up to or beyond 6 months, 239 attended their 6-month assessment visit. The disease activity and diseaserelated outcome measures for these 239 patients are summarised in Table 2. At 6 months, the mean (SD) DAS28-CRP and SDAI were 3.04 (1.25) and 11.37 (10.71), respectively (Supplementary Table 1). Based on EULAR response criterion, $110(47.2 \%)$ patients had good response, 79 (33.9\%) moderate, and $44(18.9 \%)$ no response. Regarding remission by different criteria, 97 
Table 2. Disease activity response and remission at 6 months.

\begin{tabular}{|c|c|c|c|}
\hline Characteristics & $\begin{array}{l}\text { 6-month } \\
(n=239)\end{array}$ & $\begin{array}{l}\text { 6-month change } \\
\text { from baseline }\end{array}$ & $\begin{array}{l}\text { Missing data } \\
\text { frequency }\end{array}$ \\
\hline DAS28-CRP remission (DAS28-CRP $<2.6)^{a}$ & \multicolumn{2}{|l|}{$97(41.3 \%)$} & 4 \\
\hline DAS28-ESR remission (DAS28-ESR $<2.6$ ) a & \multicolumn{2}{|l|}{$92(39.0 \%)$} & 4 \\
\hline SDAI remission (SDAI =3.3) a & \multicolumn{2}{|l|}{$57(24.3 \%)$} & 4 \\
\hline ACR/EULAR boolean remission ${ }^{a}$ & \multicolumn{2}{|l|}{$51(21.5 \%)$} & 2 \\
\hline \multicolumn{3}{|l|}{ EULAR response ${ }^{a}$} & 6 \\
\hline \multicolumn{2}{|l|}{ Good response } & \multicolumn{2}{|l|}{$110(47.2 \%)$} \\
\hline \multicolumn{2}{|l|}{ Moderate response } & \multicolumn{2}{|l|}{$79(33.9 \%)$} \\
\hline \multicolumn{2}{|l|}{ No response } & \multicolumn{2}{|l|}{$44(18.9 \%)$} \\
\hline \multicolumn{4}{|c|}{$\begin{array}{l}\text { ACR, American College of Rheumatology; DAS28-CRP and DAS28-ESR, 28-joint Disease Activity Score with C-reactive } \\
\text { protein and with erythrocyte sedimentation rate; EULAR, European League Against Rheumatism; SDAI, Simplified Disease } \\
\text { Activity Index. } \\
\text { aBased on those with outcome components not missing or where the composite outcome is inferred even if some } \\
\text { components are missing (e.g. ACR/EULAR Boolean Remission). }\end{array}$} \\
\hline
\end{tabular}

(41.3\%) patients achieved DAS28-CRP remission, 57 (24.3\%) SDAI remission, and 51 (21.5\%) met the ACR/EULAR Boolean remission criteria reflecting increased stringency of definitions. All 57 patients in SDAI remission were in DAS28CRP remission. There was excellent agreement between SDAI and ACR/EULAR Boolean remissions (Cohen's kappa of 0.9).

Other outcome measures are described in Supplementary Table 1.

\section{Predictors of clinical remission at 6 months}

Results of the univariate logistic regression models for SDAI and DAS28-CRP used for screening baseline variables for the second-stage multivariate logistic regression analyses are shown in Supplementary Table 2. Briefly, we found that sex, alcohol consumption, disease activity, HAQ, and the SF-36 Mental Component Score screened positive for both outcomes. Ethnicity screened positive for the logistic regression model for DAS28-CRP but not for SDAI. However, in the second stage, we included all variables that screened positive in the multivariate logistic regression models for either outcomes, in addition to information on prescribed RA medication.
Multivariate logistic regression models for SDAI and DAS28-CRP remissions are shown in Table 3. In both models, 6-month clinical remission was predicted by lower functional disability and disease activity at baseline. The odds ratios related to level of disease activity at baseline are 0.65 (95\% CI: $0.47-$ 0.91 ) for a 10 -unit change in SDAI or $0.67(95 \%$ CI: 0.49-0.92) for a 1-unit change in DAS28-CRP, reflecting that patients with a baseline disease activity 10 (or 1) units higher have approximately a reduction of a third in the odds of achieving 6-month remission, controlling for other variables. Higher baseline functional disability was associated with a reduced likelihood of achieving SDAI remission (odds ratio $(\mathrm{OR})=0.90,95 \% \mathrm{CI}$ : $0.83-0.98$ for a 0.125 increase in HAQ) or DAS28-CRP remission $(\mathrm{OR}=0.92,95 \% \mathrm{CI}: 0.86-0.99$ for a 0.125 increase in HAQ) at 6 months.

Although both models indicated that alcohol consumption increased the odds of remission at 6 months compared to not consuming alcohol, they gave conflicting ordering of effect sizes across the alcohol consumption categories of 1-5 units and greater than 5 units per week. Moreover, in the SDAI model, there was a suggestion that being prescribed dual combination of MTX and a second DMARD increased three-fold the likelihood 
Table 3. Multivariate logistic regression models for SDAI and DAS28-CRP remission at 6 months.

\begin{tabular}{|c|c|c|c|}
\hline Predictors & Log (odds ratio) & Standard error & $p$ value \\
\hline \multicolumn{4}{|l|}{ SDAI remission at 6 months } \\
\hline Intercept & -0.169 & 0.597 & 0.777 \\
\hline Sex (male vs female) & -0.033 & 0.409 & 0.936 \\
\hline Ethnicity (others vs white) & 0.407 & 0.439 & 0.353 \\
\hline SDAl at baseline & -0.043 & 0.017 & 0.011 \\
\hline $\mathrm{HAQ}$ at baseline & -0.818 & 0.328 & 0.013 \\
\hline SF-36 MCS at baseline & 0.025 & 0.018 & 0.167 \\
\hline Alcohol consumption at baseline & & & 0.010 \\
\hline $1-5$ units per week vs none & 1.393 & 0.491 & 0.005 \\
\hline$>5$ units per week vs none & 1.023 & 0.558 & 0.067 \\
\hline Prescribed medication at baseline & & & 0.083 \\
\hline $\begin{array}{l}\text { MTX and other DMARDs without } \\
\text { glucocorticoids vs therapies with } \\
\text { glucocorticoids }\end{array}$ & 0.392 & 0.439 & 0.372 \\
\hline $\begin{array}{l}\text { Monotherapy, not glucocorticoids vs } \\
\text { therapies with glucocorticoids }\end{array}$ & -0.706 & 0.435 & 0.105 \\
\hline \multicolumn{4}{|l|}{ DAS28-CRP remission at 6 months } \\
\hline Intercept & 1.808 & 0.768 & 0.019 \\
\hline Sex (male vs female) & -0.194 & 0.375 & 0.605 \\
\hline Ethnicity (others vs white) & -0.254 & 0.371 & 0.493 \\
\hline DAS28-CRP at baseline & -0.399 & 0.162 & 0.014 \\
\hline $\mathrm{HAQ}$ at baseline & -0.670 & 0.288 & 0.020 \\
\hline SF-36 MCS at baseline & 0.027 & 0.016 & 0.085 \\
\hline Alcohol consumption at baseline & & & 0.007 \\
\hline $1-5$ units per week vs none & 0.936 & 0.382 & 0.014 \\
\hline$>5$ units per week vs none & 1.310 & 0.456 & 0.004 \\
\hline Prescribed medication at baseline ${ }^{a}$ & & & 0.201 \\
\hline $\begin{array}{l}\text { MTX and other DMARDs without } \\
\text { glucocorticoids vs therapies with } \\
\text { glucocorticoids }\end{array}$ & 0.144 & 0.411 & 0.727 \\
\hline $\begin{array}{l}\text { Monotherapy, not glucocorticoids vs } \\
\text { therapies with glucocorticoids }\end{array}$ & -0.584 & 0.377 & 0.122 \\
\hline
\end{tabular}

HAQ, Health Assessment Questionnaire; MTX, methotrexate; SDAI, Simplified Disease Activity Index; SF-36 MCS, Short Form 36 Health Survey Mental Component Score.

aThe contrast of MTX and other DMARDs without glucocorticoids versus monotherapy gave Log (odds ratio) estimates of 1.099 and 0.728 with standard errors of 0.517 and 0.468 and $p$ values of 0.034 and 0.120 for outcomes SDAl and DAS28-CRP, respectively. 

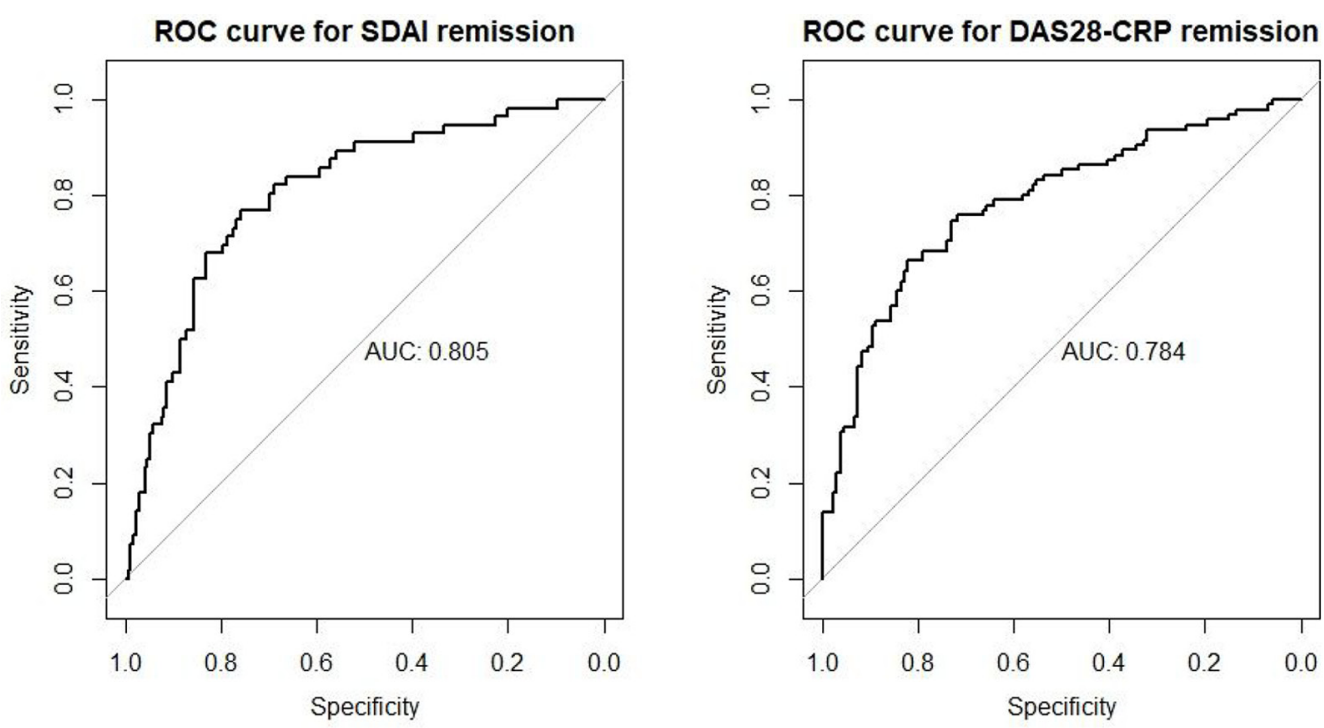

Figure 1. Receiver operating curves (ROCs) for SDAI and DAS28-CRP remission at 6 months.
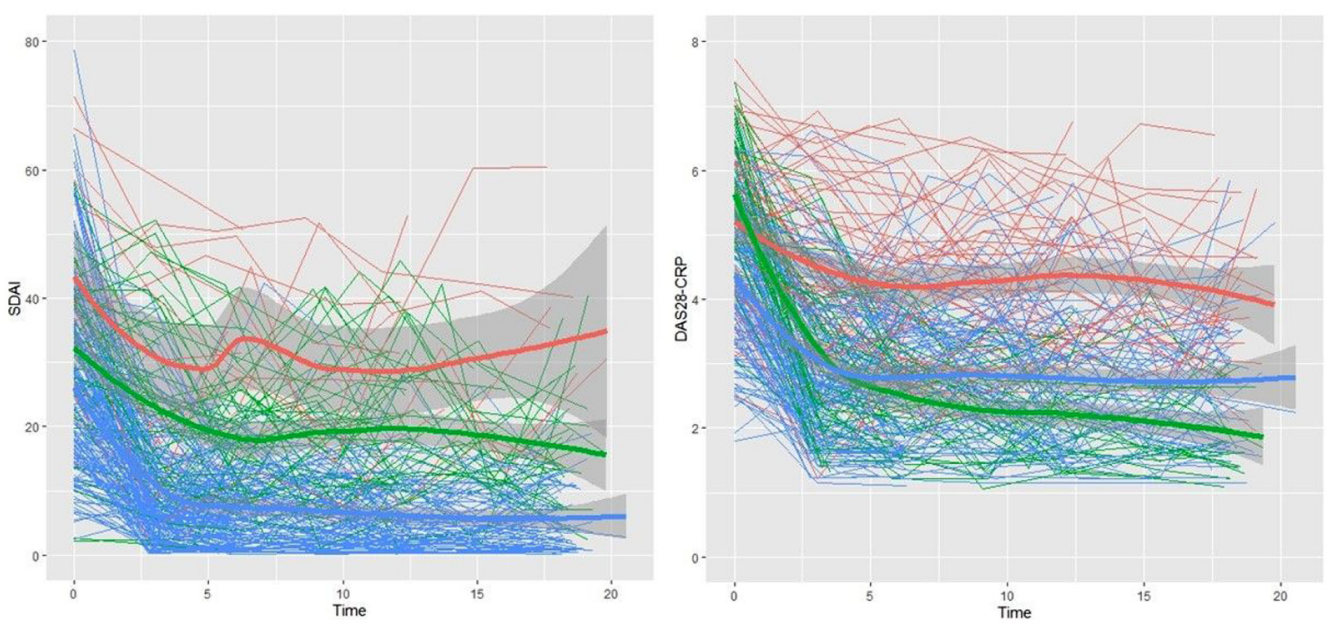

Figure 2. Individual and mean disease activity profiles over 18 months stratified by predicted class membership.

Based on SDAI, Class1 (Red; Inadequate Response (IR)): 17 (6.5\%), Class 2 (Green; higher baseline activity responder (HBAR)): 59 (22.4\%), Class 3 (Blue; lower baseline activity responder (LBAR)): 187 (71.1\%). Based on DAS28-CRP, Class1 (Red; IR): 57 (21.7\%), Class 2 (Green; HBAR): 56 (21.3\%), Class 3 (Blue; LBAR): 150 (57.0\%).

of SDAI remission compared to receiving monotherapy $\quad(\mathrm{OR}=3.00 ; \quad 95 \% \quad \mathrm{CI}=1.09-8.27$; $p=0.034)$.

The receiver operating characteristic (ROC) curves for these two multivariate logistic models for remission are shown in Figure 1. The areas under the ROC curves (AUCs) are 0.805 and 0.784 for SDAI and DAS2-CRP remission; indicating good overall performance.

\section{Characterising disease activity over time}

Figure 2 shows the observed individual trajectories of SDAI and DAS28-CRP for the 267 patients. From the figure, there is evidence of substantive within- and between-patient variation in disease activity profiles and potential distinct trajectory subtypes.

Table 4 and Supplementary Table 3 show the results of fitting LCMMs to SDAI and 
DAS28-CRP, respectively, when accounting for the baseline prescribing of medication. Both models provide evidence for three distinct subpopulations or latent classes. Class 1 corresponds to an inadequate responder (IR) group that, on average, started with high baseline disease activity that early on showed improvement, in association with initial medication, and then plateaued with moderate to high levels of disease activity. Class 2 corresponds to a higher baseline activity responder (HBAR) group that, on average, started with high baseline disease activity but showed sustained improvement over time. Class 3 corresponds to a lower baseline activity responder (LBAR) group that, on average, started with moderate levels of disease activity and showed sustained improvement (see Figure 2 and Table 5). These two three-class LCMMs had lower BIC (9368.69 and 3777.78 for three-class models vs 9421.86 and 3803.88, respectively, for four-class models) and higher relative entropy $(0.663$ and 0.591 for three-class models vs 0.621 and 0.552 ) than the corresponding four-class LCMMs.

The IR, HBAR, and LBAR groups were estimated to comprise $6.5 \%, 22.4 \%$, and $71.1 \%$ of the patients based on the SDAI model and $21.7 \%$, $21.3 \%$, and $57 \%$ of the patients based on the DAS28-CRP model. No overall statistically significant differences in mean age at entry, disease duration, SF-36 Physical Component Score at entry, sex, smoking status, alcohol consumption, and serology distributions were found across latent classes. However mean body mass index (BMI; $p=0.064)$, mean baseline levels of functional disability $(p=0.015)$, and SF-36 Mental Component Scores $(p=0.05)$ either were close to being or were statistically significantly different across the three classes/groups based on the SDAI model but not DAS28-CRP model. Table 5 shows that based on the SDAI model, the LBAR group had on average significantly less functional disability than the other groups. A similar pattern was seen for mental health, with, on average, better mental health scores seen in the LBAR group (mean of 46) compared to both IR and HBAR (means of 40.2 and 43.1, respectively). Mean BMI was approximately $3.5 \mathrm{~kg} / \mathrm{m}^{2}$ higher in the IR group compared to the other SDAI groups. No evidence was found that prescribing behaviour of clinicians varied across the SDAI or DAS28-CRP defined groups (see Supplementary Table 4).

In the IR group, lower disease activity levels over time (measured by SDAI) were associated with being female, shorter symptom duration, consuming greater than 5 units of alcohol per week, less functional disability, and better mental wellbeing at baseline. Being prescribed dual therapy of MTX and a second DMARD at baseline was associated with lower SDAI over time compared to either receiving monotherapy (excluding glucocorticoids) or therapies that included glucocorticoid usage (see Table 4).

For the HBAR group, lower levels of SDAI over time were associated with shorter symptom duration, increasing levels of alcohol consumption, and less functional disability. Moreover lower levels of disease activity were associated with being prescribed dual therapy of MTX and a second DMARD at baseline. In the LBAR group, being female, consuming alcohol and lower level of functional disability at baseline were associated with lower levels of SDAI.

Based on the DAS28-CRP model (Supplementary Table 2), lower levels of functional disability, better mental well-being and dual therapy of MTX with a second DMARD at baseline were associated with lower DAS28-CRP over time in the IR group. Higher levels of functional disability and receiving monotherapy (excluding glucocorticoids) were associated with higher levels of disease activity in the HBAR group. While being female, drinking alcohol, having lower levels of functional disability and better mental health were associated with lower DAS28-CRP over time in the LBAR group.

Table 5 summarises other patient outcome data (X-ray score, EQ-5D, HAQ, and SF-36 MCS) by allocated latent trajectory class. Class assignment could be made for 263 of the 267 eligible patients with some baseline and outcome information. The average EQ-5D scores both at baseline and 6 months were significantly higher in the LBAR group than the other groups as defined by the SDAI model $(p<0.0001$ and 0.049), while average level of functional disability remained significantly lower in this group at 6 months $(p<0.0001)$. There was no statistical evidence for a difference in average X-ray annualised progression rate across groups.

\section{Discussion}

The TACERA cohort provides a unique opportunity to follow newly diagnosed and initially medication naive patients in the United Kingdom, 
Table 4. Latent class mixed model for SDAl while controlling for baseline medication: 3 classes.

\begin{tabular}{|c|c|c|c|}
\hline & Estimate & Standard error & $p$ value \\
\hline \multicolumn{4}{|l|}{ Multinomial class-membership model } \\
\hline \multicolumn{4}{|l|}{ Class 1 (IR) vs Class 3 (LBAR) } \\
\hline Intercept & -2.118 & 0.289 & 0.000 \\
\hline \multicolumn{4}{|l|}{ Class 2 (HBAR) vs Class 3 (LBAR) } \\
\hline Intercept & -0.721 & 0.225 & 0.001 \\
\hline \multicolumn{4}{|l|}{ Linear mixed model } \\
\hline \multicolumn{4}{|l|}{ Intercept } \\
\hline Class 1 & 27.360 & 4.769 & 0.000 \\
\hline Class 2 & 15.972 & 2.593 & 0.000 \\
\hline Class 3 & 21.062 & 1.746 & 0.000 \\
\hline \multicolumn{4}{|l|}{ Disease duration, months } \\
\hline Class 1 & 1.275 & 0.526 & 0.015 \\
\hline Class 2 & 0.675 & 0.223 & 0.002 \\
\hline Class 3 & 0.136 & 0.142 & 0.339 \\
\hline \multicolumn{4}{|l|}{ Sex } \\
\hline Male vs Female Class 1 & 14.495 & 3.383 & 0.000 \\
\hline Male vs Female Class 2 & 2.039 & 1.800 & 0.257 \\
\hline Male vs Female Class 3 & 1.747 & 0.873 & 0.045 \\
\hline \multicolumn{4}{|c|}{ Alcohol consumption at baseline, units per week } \\
\hline $1-5$ units per week vs none Class 1 & -2.007 & 3.946 & 0.611 \\
\hline $1-5$ units per week vs none Class 2 & -5.420 & 1.699 & 0.001 \\
\hline $1-5$ units per week vs none Class 3 & -1.430 & 0.814 & 0.079 \\
\hline$>5$ units per week vs none Class 1 & -11.588 & 5.035 & 0.021 \\
\hline$>5$ units per week vs none Class 2 & -4.772 & 1.738 & 0.006 \\
\hline$>5$ units per week vs none Class 3 & -2.641 & 1.070 & 0.014 \\
\hline \multicolumn{4}{|l|}{ HAQ score at baseline } \\
\hline Class 1 & 5.639 & 1.417 & 0.000 \\
\hline Class 2 & 9.897 & 0.948 & 0.000 \\
\hline Class 3 & 4.070 & 0.557 & 0.000 \\
\hline \multicolumn{4}{|c|}{ Centred SF-36 Mental Component Score at baseline } \\
\hline Class 1 & -0.309 & 0.114 & 0.007 \\
\hline
\end{tabular}


Table 4. (continued)

\begin{tabular}{|c|c|c|c|}
\hline & Estimate & Standard error & $p$ value \\
\hline Class 2 & 0.090 & 0.060 & 0.133 \\
\hline Class 3 & -0.059 & 0.035 & 0.095 \\
\hline \multicolumn{4}{|c|}{ Follow-up time (in months) within 5 months } \\
\hline Class 1 & -2.456 & 0.819 & 0.003 \\
\hline Class 2 & -2.346 & 0.444 & 0.000 \\
\hline Class 3 & -4.005 & 0.260 & 0.000 \\
\hline \multicolumn{4}{|c|}{ Follow-up time (in month) after 5 months } \\
\hline Class 1 & 0.837 & 0.265 & 0.002 \\
\hline Class 2 & 0.112 & 0.145 & 0.440 \\
\hline Class 3 & 0.047 & 0.089 & 0.595 \\
\hline
\end{tabular}

Prescribed other DMARDs and MTX, not glucocorticoids vs therapies with glucocorticoids at baseline

$\begin{array}{lrrr}\text { Class } 1 & -18.742 & 2.775 & 0.000 \\ \text { Class } 2 & -7.456 & 1.775 & 0.000 \\ \text { Class 3 } & -0.838 & 1.331 & 0.529\end{array}$

Prescribed monotherapy, not glucocorticoids $v$ therapies with glucocorticoids at baseline

$\begin{array}{lrrr}\text { Class } 1 & 5.398 & 3.394 & 0.112 \\ \text { Class } 2 & -1.905 & 1.475 & 0.197 \\ \text { Class } 3 & 0.662 & 0.890 & 0.457\end{array}$

Variance components

Variance of random intercept

Variance of random slope within 5 months

Variance of random slope after 5 months

Error standard deviation
74.947

3.256

0.050

7.600
0.215

$H A Q$, Health Assessment Questionnaire; HBAR, higher baseline activity responder; IR, inadequate responder; LBAR, lower baseline activity responder; MTX, methotrexate; SDAI, Simplified Disease Activity Index; SF-36, Short Form 36 Health Survey.

whose clinical outcomes are linked to extensive and detailed biological phenotyping (to be reported separately). We focused solely on seropositive RA patients whom have few nonsevere comorbidities to reduce heterogeneity and to identify factors associated with outcomes in this subset.

We found clear evidence that remission at 6 months, whether defined using SDAI or DAS28$\mathrm{CRP}$, was associated with lower levels of disease activity and functional disability at baseline and with alcohol consumption at baseline. The findings that disease activity and functional disability are negatively associated with achieving remission have been reported before. ${ }^{28,30}$ Aletaha and colleagues $^{35}$ have previously shown that disease activity early in the course of treatment predicts response to therapy after 1 year. However, other variables previously identified as associated with remission, such as age, sex, ethnicity, and RA 
Table 5. Disease outcomes by allocated SDAl (first row of cell) and DAS28-CRP (second row of cell) classes, controlling for baseline medication.

\begin{tabular}{|c|c|c|c|c|}
\hline & Class 1 (IR) & Class 2 (HBAR) & Class3 (LBAR) & $p$ value \\
\hline \multirow[t]{2}{*}{ Baseline SDAI (SD) } & $44.65(17.50)$ & $32.08(11.99)$ & $26.44(13.66)$ & $<0.0001$ \\
\hline & $33.63(16.28)$ & $38.83(12.95)$ & $23.23(11.05)$ & $<0.0001$ \\
\hline \multirow[t]{2}{*}{ SDAl at 6 months (SD) a } & $32.84(14.10)$ & $18.74(8.97)$ & $7.36(6.82)$ & $<0.0001$ \\
\hline & $21.71(13.14)$ & $6.85(6.38)$ & $9.14(8.29)$ & $<0.0001$ \\
\hline \multirow[t]{2}{*}{ SDAI 6-month change from baseline } & $-9.11(18.70)$ & $-12.83(12.74)$ & $-19.37(14.12)$ & 0.002 \\
\hline & $-11.00(13.92)$ & $-33.28(13.94)$ & $-13.76(10.04)$ & $<0.0001$ \\
\hline \multirow[t]{2}{*}{ DAS28-CRP (baseline) } & $5.89(1.29)$ & $5.19(1.03)$ & $4.65(1.22)$ & $<0.0001$ \\
\hline & $5.22(1.24)$ & $5.73(0.89)$ & $4.38(1.11)$ & $<0.0001$ \\
\hline \multirow[t]{2}{*}{ DAS28-CRP (6 months) } & $5.12(1.23)$ & $3.99(1.02)$ & $2.58(0.93)$ & $<0.0001$ \\
\hline & $4.22(1.27)$ & $2.50(0.81)$ & $2.79(1.08)$ & $<0.0001$ \\
\hline \multirow{2}{*}{$\begin{array}{l}\text { DAS28-CRP 6-month change from } \\
\text { baseline }\end{array}$} & $-0.55(1.37)$ & $-1.19(1.15)$ & $-2.11(1.29)$ & $<0.0001$ \\
\hline & $-0.92(1.15)$ & $-3.32(1.08)$ & $-1.59(1.04)$ & $<0.0001$ \\
\hline \multirow[t]{2}{*}{ X-ray Score (baseline) } & $5.65(5.88)$ & $6.12(10.83)$ & $6.80(8.05)$ & 0.786 \\
\hline & $6.11(9.52)$ & $7.23(8.51)$ & $6.50(8.31)$ & 0.779 \\
\hline \multirow[t]{2}{*}{ X-ray annualised progression rate } & $1.00(2.30)$ & $1.08(2.86)$ & $0.89(2.61)$ & 0.898 \\
\hline & $1.39(3.64)$ & $0.79(2.04)$ & $0.80(2.32)$ & 0.372 \\
\hline \multirow[t]{2}{*}{ EQ5D Score (baseline) } & $0.33(0.38)$ & $0.43(0.31)$ & $0.52(0.32)$ & 0.030 \\
\hline & $0.43(0.33)$ & $0.45(0.34)$ & $0.52(0.32)$ & 0.094 \\
\hline \multirow[t]{2}{*}{ EQ5D Score (6 months)a } & $0.50(0.34)$ & $0.59(0.27)$ & $0.77(0.21)$ & $<0.0001$ \\
\hline & $0.63(0.27)$ & $0.79(0.18)$ & $0.72(0.25)$ & 0.003 \\
\hline \multirow[t]{2}{*}{ EQ5D 6-month change from baseline } & $0.11(0.25)$ & $0.15(0.33)$ & $0.25(0.31)$ & 0.049 \\
\hline & $0.18(0.27)$ & $0.36(0.35)$ & $0.18(0.29)$ & 0.002 \\
\hline \multirow[t]{2}{*}{ HAQ (baseline) } & $1.41(0.80)$ & $1.44(0.72)$ & $1.13(0.76)$ & 0.015 \\
\hline & $1.21(0.72)$ & $1.29(0.81)$ & $1.20(0.76)$ & 0.748 \\
\hline \multirow[t]{2}{*}{ HAQ (6 months) a } & $1.10(0.94)$ & $1.15(0.80)$ & $0.50(0.58)$ & $<0.0001$ \\
\hline & $0.93(0.83)$ & $0.50(0.61)$ & $0.65(0.68)$ & 0.008 \\
\hline \multirow[t]{2}{*}{ HAQ 6-month change from baseline } & $-0.19(0.77)$ & $-0.25(0.63)$ & $-0.63(0.60)$ & 0.0001 \\
\hline & $-0.23(0.60)$ & $-0.82(0.81)$ & $-0.52(0.52)$ & $<0.0001$ \\
\hline
\end{tabular}


Table 5. (continued)

\begin{tabular}{|c|c|c|c|c|}
\hline & Class 1 (IR) & Class 2 (HBAR) & Class3 (LBAR) & $p$ value \\
\hline \multirow[t]{2}{*}{ SF-36 MCS (baseline) } & 40.2 (11.9) & 43.1 (11.7) & 46 (11.3) & 0.05 \\
\hline & $44.4(11.4)$ & $46.5(12.2)$ & $44.6(11.3)$ & 0.632 \\
\hline \multirow[t]{2}{*}{ SF-36 MCS (6 months)a } & $41.2(10.3)$ & $46.2(13.3)$ & $50.9(10.5)$ & 0.0006 \\
\hline & $46.4(11.7)$ & $55.1(7.4)$ & $48.3(12.0)$ & 0.0002 \\
\hline \multirow{2}{*}{$\begin{array}{l}\text { SF-36 MCS 6-month change from } \\
\text { baseline }\end{array}$} & 0.15 (11.67) & $3.05(12.36)$ & $4.65(9.74)$ & 0.217 \\
\hline & 1.54 (10.68) & $7.61(10.25)$ & 3.64 (10.23) & 0.012 \\
\hline \multicolumn{5}{|c|}{$\begin{array}{l}\text { EQ5D, EuroQoL five dimensions questionnaire; HAQ, Health Assessment Questionnaire; HBAR, higher baseline activity } \\
\text { responder; IR, inadequate responder; LBAR, lower baseline activity responder; MCS, Short Form } 36 \text { Health Survey Menta } \\
\text { Component Score; SD, standard deviation; SDAI, Simplified Disease Activity Index; SF-36, Short Form } 36 \text { Health Survey. } \\
\text { Mean (SD) are reported with } p \text { values based on ANOVA test. } \\
\text { aNumber of patients at } 6 \text { months differs from the number at baseline within a class. }\end{array}$} \\
\hline
\end{tabular}

medications were not found to be statistically significant in our study. This may be due to the power of this study (especially when restricted to the outcome defined at a single time point), to the use of a treat-to-target medication strategy, or to the fact that some variables may be less important in a pure seropositive disease cohort.

We also identified three trajectories of disease course and potential factors associated with them and also with disease activity levels within trajectories. We used the same trajectory labels (IR, HBAR, and LBAR) in both SDAI and DAS28-CRP models, but the estimated proportion of patients who fell into the three groups varied according to the way disease activity was measured. Moreover, the agreement between these two classifications was weak (Cohen's Kappa of 0.18); and significant overall associations of baseline functional disability and SF-36 mental component score and near significant association with BMI were found only with the SDAI trajectories; thus suggesting these disease activity indices are not interchangeable.

Interestingly, we found no evidence for differences in prescribing behaviour at baseline among the trajectory classes irrespective of how trajectory classifications were made. However, we found clear evidence that, even as early as 6 months, disease outcomes (e.g. EQ-5D, HAQ) and their changes from baseline differed between trajectory groups, with the IR group generally having the least improvement and poorer health outcomes. No evidence for differing annualised X-ray progression rates were seen across groups. This may be expected, given the short length of follow-up and early-intensive management. The findings of very few if any significant associations between standard clinical measures and trajectory classes, evidence of clear changes in outcomes as early as 6 months and relative entropy values $(0.663$ and 0.591 ) in our latent class models that indicate further improvements can be made would possibly suggest that these trajectories may additionally be linked to distinct immunopathogenic subsets. Thus immunological biomarkers at baseline and early in follow-up (before 6 months) may be useful in identifying patients who beyond 6 months will have inadequate response to initial synthetic diseasemodifying treatment. This hypothesis is speculative at present. However, the RA-MAP Project was established towards this goal, and we are currently investigating these trajectories from a biological viewpoint, using the extensive biological data set collected in TACERA.

Heterogeneity in the effects of covariates on average SDAI level over time was seen across trajectories. Specifically, we found that effect sizes differed across trajectories with respect to functional disability, mental health, alcohol consumption, and combination MTX with second DMARD compared to therapies with glucocorticoid usage. This provides evidence to support a stratified approach to management and treatment of patients falling into different trajectory groups. For example, our models could be used to assign probabilities of different disease activity responses at 6 months or other time points to different treatment options, such as disease-modifying monotherapy, dual therapies 
without steroids, or therapies that include steroids, to decide clinically over a particular clinically relevant time horizon what would be the optimal treatment to assign a patient who presents with moderate or high levels of disease activity. In addition, the predictions of trajectory classes or the likely responses to change in treatments could be dynamically updated and refined at follow-up visits using explanatory variables and outcome information.

Our results concerning the effect of alcohol consumption on both SDAI remission at 6 months and disease activity levels within SDAI trajectories suggest that consuming alcohol is associated with higher remission rates and lower disease activity over time. Similar inverse associations have been reported by other studies. ${ }^{36-40}$ These findings may be due to alcohol intake being associated with lower levels of inflammatory markers and possibly because certain alcoholic drinks, for example, red wine, may be antioxidant due to their flavonoid content, counteracting postprandial oxidative stress. ${ }^{41,42}$

The findings that mental health is a predictor of disease activity over time in the IR group and also possibly the LBAR group but not the HBAR group are worth highlighting. One-year results from the Scottish Early Rheumatoid Arthritis Inception (SERA) cohort have identified that predictors of functional disability at 1 year appear to be dominated by psychosocial rather than more traditional clinical measures, emphasising the potential benefit which may result from early access to nonpharmacological interventions targeting key psychosocial factors, such as mental health and work disability. ${ }^{43}$ The possibility of adopting a nonpharmacological intervention strategy for those in the IR and LBAR (that make up approximately $78 \%$ of the RA patients) should be explored in more depth.

Latent class mixed modelling approaches have only recently begun to be used to investigate whether distinct subpopulations exist within RA with respect to disease course.5,44,45 Previous studies using DAS28 have identified similar trajectory groups thus suggesting that our latent classes have external validity. The larger study by Barnabe and colleagues was able to identify five trajectory groups instead of three. However, combining their Groups 1 and 4 (i.e. both with initial high activity) into a single group would coincide with our HBAR group, while combining their Groups 2 and 3 (i.e. both with initial moderate activity) would correspond to our LBAR group. In work that we have done in RA-MAP using individual participant data from multiple clinical trials, we were also able to identify three distinct latent DAS28-ESR trajectories in both baseline methotrexate-naïve treated patients and methotrexate-exposed patients. ${ }^{29}$ The baseline methotrexate-naive patients in the trials reflected a relatively early in disease population which is more comparable to our TACERA cohort. It thus was reassuring that similar latent trajectory classes were identified from TACERA using both DAS28-CRP and SDAI. Our work therefore demonstrates that similar trajectory groups exist within a purely seropositive population with low levels of comorbidities. In addition, we controlled for factors that may impact on disease activity over time within these trajectory groups.

The decision to restrict to an inception cohort with low levels of comorbidities was made to reduce variation due to comorbidities when addressing the RA-MAP Project's primary objective of understanding immune function and response in RA through use of an extensive set of biological markers, measured at baseline and over time. This decision has implications for generalisability to the newly diagnosed RA population in the United Kingdom, especially those with significant comorbidities.

\section{Conclusion}

In conclusion, we found that in early seropositive RA with few nonsevere comorbidities, lower baseline levels of functional disability, and disease activity, along with alcohol intake, are associated with 6-month clinical remission. We identified three subpopulations based on disease trajectories that not only differ in terms of disease course but also with regard to the effect of risk factors, such as mental well-being, on disease activity over time. Our data further highlight RA heterogeneity (i.e. trajectory classes) not explainable by clinical factors and indicate the possible use of biomarkers collected at baseline and early follow-up to help patient management and better targeting of existing and novel therapies.

\section{Acknowledgements}

The author acknowledges the support of TACERA Principal Investigators from all contributing NHS sites and the members of the TACERA Study Steering and Data Monitoring Committee. This research was supported by the NIHR Newcastle 
Biomedical Research Centre and the NIHR Cambridge Biomedical Research Centre. (The views expressed are those of the author(s) and not necessarily those of the NHS, the NIHR or the Department of Health and Social Care) Members of the RA-MAP Consortium can be found in the supplementary document "RA-MAP Consortium Membership.docx".

\section{The RA-MAP Consortium}

Contributing authors: Michael Barnes (Queen Mary University of London, London, UK), Sarah Brockbank (Newcastle University, Newcastle upon Tyne, UK), Ian N Bruce (Manchester Academic Health Science Centre, Manchester, UK), Coziana Ciurtin (University College London Hospital, London, UK), Andrew P. Cope (King's College London, London, UK), Michael R. Ehrenstein (UCL, London, UK), Paul Emery (University of Leeds, Leeds, UK), Benjamin A. Fisher (University of Birmingham, Birmingham, UK), John Isaacs (Newcastle University, Newcastle upon Tyne, UK), Ruth Matthews (The Institute of Cancer Research, Sutton, UK), Iain B. McInnes (University of Glasgow, Glasgow, UK), Hayley Noble (University of London, London, UK), Ayako Wakatsuki Pedersen (Newcastle University, Newcastle upon Tyne, UK), Costantino Pitzalis (Queen Mary University of London, London, UK), Karim Raza (University of Birmingham, Birmingham, UK), Anthony Rowe (Janssen Research \& Development, Pennsylvania, USA), Gemma Simpson (King's College London, London, UK), Dominic Stringer (King's College London, London, UK), Peter C. Taylor (University of Oxford, Oxford, UK), Brian Tom (University of Cambridge, Cambridge, UK), Yujie Zhong (University of Cambridge, Cambridge, UK).

\section{Author contributions}

A list of contributing authors with details of contributions can be found in the supplementary document "RA-MAP Contributing Authors.docx".

\section{Conflict of interest statement}

A list of contributing authors with disclosure of possible conflicts of interests can be found in the supplementary file "RA-MAP Contributing Authors.docx".

\section{Funding}

The authors disclosed receipt of the following financial support for the research, authorship, and/or publication of this article: This study was funded by the MRC/ABPI Inflammation and Immunology Initiative Grant (MRC reference numbers: G1001516 and G1001518). Dr Brian Tom is supported by the UK Medical Research Council (Unit Programme number $\mathrm{MC}_{-}$ UP_1302/3 and MC_UU_00002/2).

\section{Ethical approval and consent to participate}

Ethical approval was authorised by the National Research Ethics Service London Central Committee (reference number: 12/LO/0469). Informed, written consent was obtained.

\section{ORCID iD}

Brian Tom iD https://orcid.org/0000-0002-33359322

\section{Availability of data and materials}

Request for access to RA-MAP data and samples can be made to the RA-MAP Project Manager (https://research.ncl.ac.uk/ra-map/ra-mapoutputs/).

\section{Supplemental material}

Supplemental material for this article is available online.

\section{References}

1. Scott DL, Wolfe F and Huizinga TW. Rheum arthritis. Lancet 2010; 376: 1094-1108.

2. Cook MJ, Diffin J, Scire CA, et al. Predictors and outcomes of sustained, intermittent or never achieving remission in patients with recent onset inflammatory polyarthritis: results from the Norfolk Arthritis Register. Rheumatology 2016; 55: 1601-1609.

3. Ma MHY, Ibrahim F, Kingsley GH, et al. Variable impacts of different remission states on health-related quality of life in rheumatoid arthritis. Clin Exp Rheumatol 2018; 36: 203-209.

4. Wolfe F, Rasker JJ, Boers M, et al. Minimal disease activity, remission, and the long-term outcomes of rheumatoid arthritis. Arthritis Care Res 2007; 57: 935-942.

5. Siemons L, Ten Klooster PM, Vonkeman HE, et al. Distinct trajectories of disease activity over the first year in early rheumatoid arthritis patients following a treat-to-target strategy. Arthritis Care Res 2014; 66: 625-630.

6. Norton S, Fu B, Scott DL, et al. Health Assessment Questionnaire disability progression in early rheumatoid arthritis: systematic review 
and analysis of two inception cohorts. Semin Arthritis Rheum 2014; 44: 131-144.

7. Bukhari M, Thomson W, Naseem H, et al. The performance of anti-cyclic citrullinated peptide antibodies in predicting the severity of radiologic damage in inflammatory polyarthritis: results from the Norfolk Arthritis Register. Arthritis Rheum 2007; 56: 2929-2935.

8. van Gaalen FA, van Aken J, Huizinga TW, et al. Association between HLA class II genes and autoantibodies to cyclic citrullinated peptides (CCPs) influences the severity of rheumatoid arthritis. Arthritis Rheum 2004; 50: 2113-2121.

9. Cope AP, Barnes MR, Belson A, et al. The RA-MAP Consortium: a working model for academia-industry collaboration. Nat Rev Rheumatol 2018; 14: 53-60.

10. Aletaha D, Neogi T, Silman AJ, et al. 2010 rheumatoid arthritis classification criteria: an American College of Rheumatology/European League Against Rheumatism collaborative initiative. Arthritis Rheum 2010; 62: 2569-2581.

11. Arnett FC, Edworthy SM, Bloch DA, et al. The American Rheumatism Association 1987 revised criteria for the classification of rheumatoid arthritis. Arthritis Rheum 1988; 31: 315-324.

12. NICE. The management of rheumatoid arthritis in adults (Clinical guideline 79). London: NICE, 2009, www.nice.org.uk/CG79

13. Aletaha D and Smolen J. The Simplified Disease Activity Index (SDAI) and the Clinical Disease Activity Index (CDAI): a review of their usefulness and validity in rheumatoid arthritis. Clin Exp Rheumatol 2005; 23(5 Suppl. 39): S100-S108.

14. Anderson J, Caplan L, Yazdany J, et al. Rheumatoid arthritis disease activity measures: American College of Rheumatology recommendations for use in clinical practice. Arthritis Care Res 2012; 64: 640-647.

15. Anderson JK, Zimmerman L, Caplan L, et al. Measures of rheumatoid arthritis disease activity: Patient (PtGA) and Provider (PrGA) Global Assessment of Disease Activity, Disease Activity Score (DAS) and Disease Activity Score with 28-Joint Counts (DAS28), Simplified Disease Activity Index (SDAI), Clinical Disease Activity Index (CDAI), Patient Activity Score (PAS) and Patient Activity Score-II (PASII), Routine Assessment of Patient Index Data (RAPID), Rheumatoid Arthritis Disease Activity Index (RADAI) and Rheumatoid Arthritis Disease Activity Index-5 (RADAI-5), Chronic Arthritis Systemic Index (CASI), Patient-Based Disease Activity Score With ESR (PDAS1) and
Patient-Based Disease Activity Score without ESR (PDAS2), and Mean Overall Index for Rheumatoid Arthritis (MOI-RA). Arthritis Care Res 2011; 63(Suppl. 11): S14-S36.

16. Dougados M, Aletaha D and van Riel P. Disease activity measures for rheumatoid arthritis. Clin Exp Rheumatol 2007; 25: S22-S29.

17. Felson DT, Smolen JS, Wells G, et al. American College of Rheumatology/European League Against Rheumatism provisional definition of remission in rheumatoid arthritis for clinical trials. Arthritis Rheum 2011; 63: 573-586.

18. Larsen A. A radiological method for grading the severity of rheumatoid arthritis. Scand $\mathcal{F}$ Rheumatol 1975; 4: 225-233.

19. Larsen A, Dale K and Eek M. Radiographic evaluation of rheumatoid arthritis and related conditions by standard reference films. Acta Radiol Diagn 1977; 18: 481-491.

20. Scott DL, Houssien DA and Laasonen L. Proposed modification to Larsen's scoring methods for hand and wrist radiographs. $\mathrm{Br} \mathcal{F}$ Rheumatol 1995; 34: 56.

21. Fries JF, Spitz P, Kraines RG, et al. Measurement of patient outcome in arthritis. Arthritis Rheum 1980; 23: 137-145.

22. Hurst NP, Kind P, Ruta D, et al. Measuring health-related quality of life in rheumatoid arthritis: validity, responsiveness and reliability of EuroQol (EQ-5D). BrF Rheumatol 1997; 36: 551-559.

23. Linde L, Sørensen J, Østergaard M, et al. Health-related quality of life: validity, reliability, and responsiveness of SF-36, EQ-15D, EQ-5D, RAQoL, and HAQ in patients with rheumatoid arthritis. F Rheumatol 2008; 35: 1528-1537.

24. Ruta DA, Hurst NP, Kind P, et al. Measuring health status in British patients with rheumatoid arthritis: reliability, validity and responsiveness of the short form 36-item health survey (SF-36). $\mathrm{Br}$ F Rheumatol 1998; 37: 425-436.

25. Forslind K, Hafström I, Ahlmen M, et al. Sex: a major predictor of remission in early rheumatoid arthritis? Ann Rheum Dis 2007; 66: 46-52.

26. Katchamart W, Johnson S, Lin HJ, et al. Predictors for remission in rheumatoid arthritis patients: a systematic review. Arthritis Care Res 2010; 62: 1128-1143.

27. Furst DE, Pangan AL, Harrold LR, et al. Greater likelihood of remission in rheumatoid arthritis patients treated earlier in the disease course: results from the Consortium of Rheumatology Researchers of North America registry. Arthritis Care Res 2011; 63: 856-864. 
28. Castrejón I, Dougados M, Combe B, et al. Prediction of remission in a French early arthritis cohort by RAPID3 and other core data set measures, but not by the absence of rheumatoid factor, anticitrullinated protein antibodies, or radiographic erosions. $\mathcal{F}$ Rheumatol 2016; 43 : 1285-1291.

29. RA-MAP Consortium. Novel methodology to discern predictors of remission and patterns of disease activity over time using rheumatoid arthritis clinical trials data. RMD Open 2018; 4: e000721.

30. Baganz L, Richter A, Albrecht K, et al. Are prognostic factors adequately selected to guide treatment decisions in patients with rheumatoid arthritis? A collaborative analysis from three observational cohorts. Semin Arthritis Rheum 2019; 48: 976-982.

31. R Core Team. R: a language and environment for statistical computing (Computer software). Vienna: R Foundation for Statistical Computing, 2017.

32. Vandenbroucke JP, Von Elm E, Altman DG, et al. Strengthening the Reporting of Observational Studies in Epidemiology (STROBE): explanation and elaboration. PLoS Med 2007; 4: e297.

33. Charlson ME, Pompei P, Ales KL, et al. A new method of classifying prognostic comorbidity in longitudinal studies: development and validation. J Chronic Dis 1987; 40: 373-383.

34. Charlson ME, Charlson RE, Peterson JC, et al. The Charlson Comorbidity Index is adapted to predict costs of chronic disease in primary care patients. F Clin Epidemiol 2008; 61: 1234-1240.

35. Aletaha D, Funovits J, Keystone EC, et al. Disease activity early in the course of treatment predicts response to therapy after one year in rheumatoid arthritis patients. Arthritis Rheum 2007; 56: 3226-3235.

36. Dunlop DD, Semanik P, Song J, et al. Risk factors for functional decline in older adults with arthritis. Arthritis Rheum 2005; 52: 1274-1282.
37. Kim S-K, Park S-H, Shin I-H, et al. Anti-cyclic citrullinated peptide antibody, smoking, alcohol consumption, and disease duration as risk factors for extraarticular manifestations in Korean patients with rheumatoid arthritis. $\mathcal{F}$ Rheumatol 2008; 35: 995-1001.

38. Maxwell JR, Gowers IR, Moore DJ, et al. Alcohol consumption is inversely associated with risk and severity of rheumatoid arthritis. Rheumatology 2010; 49: 2140-2146.

39. Nissen MJ, Gabay C, Scherer A, et al. The effect of alcohol on radiographic progression in rheumatoid arthritis. Arthritis Rheum 2010; 62: 1265-1272.

40. Papadopoulos NG, Alamanos Y, Voulgari $\mathrm{PV}$, et al. Does cigarette smoking influence disease expression, activity and severity in early rheumatoid arthritis patients? Clin Exp Rheumatol 2005; 23: 861-866.

41. Covas MI, Gambert P, Fitó M, et al. Wine and oxidative stress: up-to-date evidence of the effects of moderate wine consumption on oxidative damage in humans. Atherosclerosis 2010; 208: 297-304.

42. Lu B, Solomon DH, Costenbader KH, et al. Alcohol consumption and markers of inflammation in women with preclinical rheumatoid arthritis. Arthritis Rheum 2010; 62: 3554-3559.

43. Kronisch C, McLernon DJ, Dale J, et al. Brief report: predicting functional disability: one-year results from the Scottish Early Rheumatoid Arthritis inception cohort. Arthritis Rheumatol 2016; 68: 1596-1602.

44. Barnabe C, Sun Y, Boire G, et al. Heterogeneous disease trajectories explain variable radiographic, function and quality of life outcomes in the Canadian Early Arthritis Cohort (CATCH). PLoS ONE 2015; 10: e0135327.

45. Wabe N, Sorich MJ, Wechalekar MD, et al. Characterising deviation from treat-to-target strategies for early rheumatoid arthritis: the first three years. Arthritis Res Ther 2015; 17: 48.
Visit SAGE journals online journals.sagepub.com/ home/tab

@SAGE journals 